\title{
Stress Detection using Wearable Physiological Sensors
}

\author{
Virginia Sandulescu ${ }^{1}$, Sally Andrews ${ }^{2}$, David Ellis ${ }^{2}$, Nicola Bellotto ${ }^{2}$, and \\ Oscar Martinez Mozos ${ }^{2}$ \\ 1 Politehnica University of Bucharest, Bucharest, Romania, \\ S_virg@yahoo.com \\ 2 University of Lincoln, Lincoln, UK \\ s.andrews@lincoln.ac.uk, dellis@lincoln.ac.uk, nbellotto@lincoln.ac.uk, \\ omozos@lincoln.ac.uk
}

\begin{abstract}
As the population increases in the world, the ratio of health carers is rapidly decreasing. Therefore, there is an urgent need to create new technologies to monitor the physical and mental health of people during their daily life. In particular, negative mental states like depression and anxiety are big problems in modern societies, usually due to stressful situations during everyday activities including work. This paper presents a machine learning approach for stress detection on people using wearable physiological sensors with the final aim of improving their quality of life. The presented technique can monitor the state of the subject continuously and classify it into "stressful" or "non-stressful" situations. Our classification results show that this method is a good starting point towards real-time stress detection.
\end{abstract}

Keywords: stress detection, wearable physiological sensors, assistive technologies, signal classification, quality of life technologies

\section{Introduction}

As the population increases in the world, the ratio of health carers is rapidly decreasing. Actually, the Organisation for Economic Co-operation and Development (OECD) warns about future shortages of available health workers and doctors [3]. Therefore, there is an urgent need to create new technologies to monitor the health of people, both physical and mental, during their daily life with the aim of supporting health workers, caregivers, and doctors in their tasks. These technologies, also known as Quality of Life Technologies (QoLTs), have emerged as the concept of applying findings from different technological areas to assist people and improve their quality of life.

An emerging research topic inside QoLTs is their application to psychology and self-therapy to improve the mood of people and thus, their quality of life. Although there exist several technologies to support the health of people at the physiological level, the technologies that are able to provide similar support at the mental level are almost inexistent. 
Treating negative mental states in people is becoming a priority in our new societies. In particular, stress is a big problem in modern populations due to the increment of stressful situations during everyday activities including work. Stress is a natural reaction of the human body to an outside perturbing factor. The physiological responses to stress are correlated with variations in heart rate, blood volume pulse, skin temperature, pupil dilation, electro-dermal activity $[18,17,13]$. Stress may have beneficial effects on fighting the stress factor, like increasing reflexes, but it was determined that long term stress is correlated with various health problems like depression and premature ageing [16], [9].

Stress is creating new problems that have a great impact in our societies and economies. For example, according to the Mental Health Foundation in UK [2], around 12 million adults in the UK visit their general practitioner doctor (GP) each year with mental health problems, most of which are related to stress. As a consequence, 13.3 million working days are lost per year due to stress problems. Moreover, according to the World Health Organization [4], stress has a cost of around 8.4 million to UK enterprises. Finally, current appointments for national health mental services in UK, such as Cognitive Behavioural Therapy (CBT) [5] are taking 3-6 months to be processed, with the subsequent danger for the patient because cumulative stress may have broad negative consequences on societal well-being and costs [15]. Thus, the research of this paper emerges as a necessity to create new wearable technologies to monitor stress on people during their daily life.

This paper presents a machine learning approach for stress detection on people using wearable physiological sensors with the final aim of improving their quality of life. Moreover, the presented technique monitors the state of the subject continuously and classifies it into "stressful" or "non-stressful" situations. Finally, our classification results shows that our approach is a good starting point towards real-time stress detection and treatment.

\section{Wearable Physiological Sensors}

In this paper we aim to detect stress in people using wearable sensors that measure physiological responses. In particular, we have used the BioNomadix module from Biopac, model BN-PPGED [1] as shown in Figure 1.

The BN-PPGED is worn as a wristband on the non-dominant hand of a subject with two electrodes situated on two fingers that measure the electro-dermal activity (EDA) and the pulse plethysmograph (PPG) signals. EDA, sometimes measured as electrodermal response, skin conductance activity, or galvanic skin response, is an indication of skin sweating activity. PPG, also known as Blood Volume Pulse (BVP), is obtained using a pulse oxiometer which illuminates the skin and measures the differences in light absorption. The amount of light that returns to the PPG sensor is proportional to the volume of blood in the tissue [14].

In our experiments the EDA and PPG physiological signals were acquired at a $1000 \mathrm{~Hz}$ sampling frequency. After the acquisition the signals were down- 


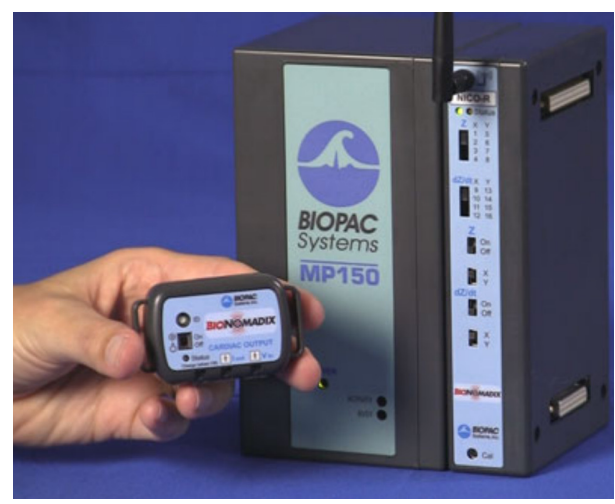

Fig. 1. BioNomadix model BN-PPGED and MP150 station by biopac [1].

sampled to $10 \mathrm{~Hz}$. Afterwards, a filtering and artefact removal approach was applied by using the routines included in the AcqKnowledge software [1]. In adittion, AcqKnowledge was used to extract the PPG autocorrelation signal and the Heart Rate Variability (HRV). HRV represents the beat-to-beat variability over a given period of time and is computed by calculating the standard deviation of the average of normal-to-normal heartbeats [14].

The BN-PPGED connects though wireless to a Biopac MP150 communication station as shown in Figure 1. The MP150 station directly connects to a computer that runs AcqKnowledge 4 software for real-time data acquisition [1]. In this way, the subject wearing the sensors can move freely while the experiments and the different signals are send through wireless to a computer.

\section{Classification of Physiological Signals}

In our approach we classify the state of each person at 0.1 seconds intervals. Each state is composed of four measurements: PPG value $(p p g)$, PPG autocorrelation value (ppgau), HRV value ( $h r v)$, and EDA value (eda). Thus, we represent each sample at time $t$ as the feature vector $x_{t}=\left\{p p g_{t}, p p g a u_{t}, h r v_{t}, e d a_{t}\right\}$, where $t$ is sampled at 0.1 seconds intervals.

Each sample $x_{t}$ was labelled according to the state of the person at that time, i.e. stressed, or not stressed. Thus, our dataset was composed of the measurements obtained at each time interval together with their corresponding label as $D=\left\{\left(x_{t}, l_{t}\right)\right\}$, with $l_{t} \in L=\{$ stressed,not_stressed $\}$. The state of the person $l_{t}$ was defined by the activity that person was performing at time $t$ during the experiment (see Section 4).

The classification of the sampled meassurements was done using a support vector machine (SVM) [8,6]. Support vector machines take as input a set of $n$ feature vectors $x_{i}$ together with their labels $y_{i} \in Y=\{1,-1\}$. The idea behind SVMs is to find the hyperplane that maximizes the distance between the 
examples of the two classes $\{1,-1\}$. This is done by finding a solution to the optimization problem

$$
\min _{w, b, \xi} C \sum_{i=1}^{n} \xi_{i}+\frac{1}{2}\|w\|^{2},
$$

subject to the condition

$$
y_{i}\left(w^{T} \phi\left(\boldsymbol{x}_{\boldsymbol{i}}\right)+b\right) \geq 1-\xi_{i},
$$

where $w$ is the normal to the hyperplane, and $\xi_{i} \geq 0$ are slack variables that measure the error in the misclassification of $x_{i}$. In addition, we use a radial basis function (RBF) kernel

$$
K\left(x_{i}, x_{j}\right)=\exp \left(-\gamma\left\|x_{i}-x_{j}\right\|^{2}\right), \gamma>0
$$

In our case, we map our original labels $L=\{$ stressed,not_stressed $\}$ into $Y=\{1,-1\}$ so that our examples could be used in a SVM.

\section{Experimental Setup}

To check the validity of our stress detector we prepared an experimental setup where different subjects experimented different stressful situations. In this section we will describe the complete experimental setup and protocol.

In the study presented in [9], more than 200 stress experiments are reviewed in terms of activities involved in the experiments and the cortisol responses measured on the subjects performing these activities. According to the same source, the most effective tasks for inducing stress are public speaking and cognitive tasks, because during these tasks the highest increases in cortisol levels are measured. This is why our designed experiment contained both a public speaking task and a cognitive task.

Our final designed experiment is based on the Trier Social Stress Test (TSST) [12]. This is a very popular experimental setup and it has been used in more than 4000 sessions during the last decades [9]. The TSST consists of a neutral task followed by a public speaking task, a cognitive task and another neutral task in the end. Each neutral task consists of 2 minutes of predefined neutral questions like: "How do you find the weather today" or "How did you get here?". The public speaking is a 5 minute interview for a desired job. After this, the participant is asked to count back in steps of 13, starting from 1022. This is the cognitive task. All the previous tasks are performed in front of a live audience and a video camera. The camera is only used to induce the stress more reliably [9], so the recordings are not stored. The neutral tasks are thought as non-stressful situations, while the speaking and cognitive tasks are considered stressful situations.

In more detail, our protocol for the TSST was as follows. When the participants enter the experiment room, they are given verbal and written information about the procedures involved in the experiment. The participants are asked to 
fill in a consent form and to confirm that they do not suffer from any cardiovascular or anxiety disorder that might be affected by experiencing stress or that might affect the results of the experiment.

After being briefed, the participants are asked to fill in a State Trait Anxiety Inventory (STAI) [10] to estimate the current level of stress. They are then fitted with the sensors. There is a 2 minutes period of time when the participants are asked predefined neutral questions, in order to determine the baseline, which we will used as neutral state. Afterwards, the participants are asked to sit at a desk and prepare a presentation for an job interview job during 3 minutes. They are given a pen and a paper for this. When the 3 minutes time expires, they are asked to hand out the sheet of paper and stand up in a predefined square on the ground and begin their presentation. During the 5 minutes of the presentation, the participants are encouraged to speak continuously. If the participants stop during the presentation, at the first pause, they are told about the remaining time and asked to continue. At the next pause, they are asked a set of predefined typical interview questions like: "What are your strengths/weaknesses?", "Where do you see yourself in 5 years?" and so on. After the 5 minutes presentations. The participants are explained a cognitive task and the 5 minutes timer is started. Whenever the participants say the wrong number, they are asked to start again from 1022. At the end of the cognitive task, the participants are given a short time to relax, while given another debrief. Then another two minutes of neutral questions are recorded. Finally, the participants are then asked to fill in the STAI questionnaire to estimate the current level of stress and the general level of stress.

\section{Experimental Results}

The previous TSST session was conducted on 5 participants $\{\mathrm{P} 1, \mathrm{P} 2, \mathrm{P} 3, \mathrm{P} 4$, P5\}, that were volunteering students from the School of Psychology, at the University of Lincoln, aged 18 to 39, both males and females.

The goal of these experiments is to check the performance of our approach to create a personalized stress detector for each participant. For this reason we created independent datasets of measurements for each participant $\mathcal{D}=$ $\left\{D_{1}, D_{2}, D_{3}, D_{4}, D_{5}\right\}$. The size of datasets $D_{k}$, i.e. number of feature vectors (c.f. Section 3), were $\left|D_{1}\right|=11620,\left|D_{2}\right|=13450,\left|D_{3}\right|=13740,\left|D_{4}\right|=13740$, and $\left|D_{5}\right|=13000$.

We trained a $\mathrm{SVM}_{k}$ for each participant using the corresponding dataset $D_{k}$ and evaluated the classifier according to it. For each $\mathrm{SVM}_{k}$ we used $75 \%$ of the corresponding dataset $D_{k}$ for training and the remaining $25 \%$ for testing. To create this sets we used a stratified selection to ensure the same class distribution in the subset as in the original set. Then the training and set data were scaled to have values in the $[-1,1]$ range. 
In our experiments we used the LIBSVM library [7]. Moreover, following the method in [11], the parameters $C$ and $\gamma$ for each $\mathrm{SVM}_{k}$ were selected by grid-search using cross-validation.

The results of the different detectors are shown in Table 1. We can see that we obtain very good detection results in all the participants, with accuracies over $82 \%$ in two cases, and precissions over $80 \%$ in the majority of the patients.

Table 1. Classification accuracy and precision

\begin{tabular}{ccc}
\hline Participant No. Accuracy & [\%] & Precision [\%] \\
\hline P1 & 78.90 & 80.19 \\
P2 & 73.26 & 73.61 \\
P3 & 83.08 & 83.87 \\
P4 & 82.82 & 83.20 \\
P5 & 76.83 & 76.67 \\
\hline
\end{tabular}

The individual confusion matrices for each participant are shown in Table 2. The results suggest a bias to classify non-stressful states as stressed. We think this is due to the fact that people remained stress during short periods of times during the transitions to neutral tasks, since they need time to relax. However, this transition time was not taken into account in these results.

Table 2. Individual confusion matrices

\begin{tabular}{|c|c|c|c|c|c|c|c|c|c|c|}
\hline \multirow[t]{2}{*}{ Participant No. } & \multicolumn{2}{|c|}{$\mathrm{P} 1$} & \multicolumn{2}{|c|}{$\mathrm{P} 2$} & \multicolumn{2}{|c|}{ P3 } & \multicolumn{2}{|c|}{$\mathrm{P} 4$} & \multicolumn{2}{|c|}{ P5 } \\
\hline & 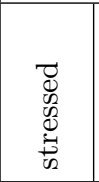 & 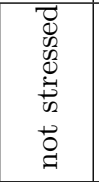 & 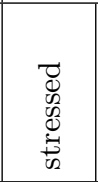 & 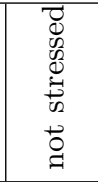 & 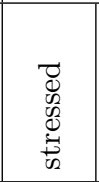 & 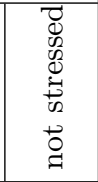 & 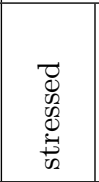 & 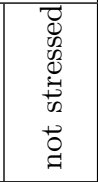 & 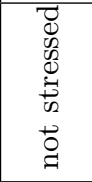 & 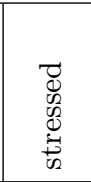 \\
\hline stressed & 94.04 & 5.95 & 88.21 & 11.79 & 90.50 & 9.50 & 91.55 & 8.44 & 91.62 & 8.38 \\
\hline not stressed & 60.62 & 39.38 & 50.73 & 49.27 & \begin{tabular}{|l|}
29.49 \\
\end{tabular} & 70.51 & 32.53 & 67.47 & 49.36 & 50.64 \\
\hline
\end{tabular}

\section{Conclusions}

In this paper we have presented an approach for stress detection using wearable physiological sensors. Our approach is able to analyse the state of the subject at any instant an decide about his/her stress situation. Detection results in our 
experiment demonstrate that our approach is a good starting point towards realtime mental mood detection and treatment on people to improve their quality of life.

\section{References}

1. Biopac. http://www.biopac.com.

2. Mental Health Foundation in UK. http://www.mentalhealth.org.uk.

3. The Organisation for Economic Co-operation and Development (OECD). http://www.oecd.org.

4. World Health Organization (WHO). http://www.who.org.

5. A.T. Beck. Cognitive therapy and the emotional disorders. International Universities Press, Inc., Madison, CT, 1975.

6. Christopher M. Bishop. Pattern Recognition and Machine Learning. Springer, 2006.

7. Chih-Chung Chang and Chih-Jen Lin. LIBSVM: A library for support vector machines. ACM Transactions on Intelligent Systems and Technology, 2:27:1-27:27, 2011. Software available at http://www.csie.ntu.edu.tw/ cjlin/libsvm.

8. C. Cortes and V. Vapnik. Support-vector network. Machine Learning, 20:273-297, 1995.

9. S. S. Dickerson and M. E. Kemeny. Acute stressors and cortisol responses: A theoretical integration and synthesis of laboratory research. Psychological Bulletin, 130(3):355-391, 2004.

10. S. Elwood, L., K. Wolitzky-Taylor, and O. Olatunji, B. Measurement of anxious traits: a contemporary review and synthesis. Anxiety Stress Coping, 25(6):647-66, 2012.

11. Chih-Wei Hsu, Chih-Chung Chang, and Chih-Jen Lin. A practical guide to support vector classification. http://www.csie.ntu.edu.tw/ cjlin/papers/guide/guide.pdf, 2010.

12. C. Kirschbaum, K. M. Pirke, and D. H. Hellhammer. The T́rier Social Stress Test-A tool for investigating psychobiological stress responses in a laboratory setting. Neuropsychobiology, pages 76-81, 1993.

13. Wikgren M., Maripuu M., Karlsson T., Nordfjäll K., Bergdahl J., Hultdin J., DelFavero J., Roos G., Nilsson L.G., Adolfsson R., and Norrback K.F. Short telomeres in depression and the general population are associated with a hypocortisolemic state. Biological Psychiatry, 71(4):294-30, 2012.

14. E. Peper, R. Harvey, I-Mei L., H. Tylova, and D. Moss. Is there more to blood volume pulse than heart rate variability, respiratory sinus arrhythmia, and cardiorespiratory synchrony? Biofeedback, 35(2):54-61, 2007.

15. A. Perkins. Saving money by reducing stress. Harvard Business Review, 72(12), 1994.

16. D. Rai, K. Kosidou, M. Lundberg, R. Araya, G. Lewis, and C. Magnusson. Psychological distress and risk of long-term disability: population-based longitudinal study. Journal of Epidemiology and Community Health, 66(7):586-92, 2011.

17. F. T. Sun, C. Kuo, H. T. Cheng, B. Senaka, P. Collins, and M. Griss. Activity-aware mental stress detection using physiological sensors. Lecture Notes of the Institute for Computer Sciences, Social Informatics and Telecommunications Engineering, 76:211-230, 2012.

18. M. Sung and A. Pentland. PokerMetrics: Stress and Lie Detection through Noninvasive Physiological Sensing. PhD thesis, MIT Media Laboratory, 2005. 